\title{
The nuclear data program at rensselaer
}

\author{
Y. Danon ${ }^{1}$, Z. Blain ${ }^{1}$, R. Bahran ${ }^{1}$, M. Rapp ${ }^{1}$, J. Thompson ${ }^{1}$, D. Williams ${ }^{1}$, G. Leinweber ${ }^{2}$, D. Barry ${ }^{2}$, \\ R. Block ${ }^{2}$, and J. Hoole ${ }^{2}$ \\ 1 Rensselaer Polytechnic Institute, Troy, NY 12180, USA \\ 2 Bechtel Marine Propulsion Corporation, Knolls Atomic Power Laboratory, PO Box 1072, \\ Schenectady, New York 12301-1072, USA
}

\begin{abstract}
The nuclear data program at the Rensselaer Polytechnic Institute (RPI) is centered around a $60 \mathrm{MeV}$ pulsed electron Linear Accelerator (LINAC) configured to deliver pulsed neutron beams. The LINAC electron pulse width can vary between $5 \mathrm{~ns}$ and 5 us, a repetition rate of 1-500 pulses per second and neutron yield of up to $1013 \mathrm{n} / \mathrm{s}$. Over the years several experimental setups and techniques were developed to enable a variety of measurement capabilities. The experiments cover the energy range from $0.001 \mathrm{eV}$ to $20 \mathrm{MeV}$ and included neutron transmission, capture, scattering and fission measurements. The facility is also equipped with a Lead Slowing-Down Spectrometer (LSDS) producing a high neutron flux that is used for simultaneous measurements of the fission cross section and fission fragment mass and energy distributions. Detectors for (n, alpha) and (n, p) cross section measurements using the LSDS were also developed and measurements of (n, alpha) cross section were completed. The high neutron flux inside the LSDS enables measurements on small samples (sub micrograms) or samples with small cross section (sub millibarns).

In order to fully utilize the capabilities of the facility, several measurement techniques were developed; high accuracy $(<1 \%)$ total cross sections were measured using iron and uranium filtered neutron beams. A system for fast neutron scattering measurements using an array of liquid scintillators and a digital data acquisition system was recently developed and used for several measurements and is now being adapted for fission neutron studies. Methods for simultaneous measurements of fission and capture cross sections using the RPI multiplicity detector are under development and new modular detectors for both fast and resonance region neutron detection were developed and deployed.

These developments result in numerous measurements on different materials and provide input to improved evaluated nuclear data libraries and thus help improve the accuracy of calculations of nuclear reactors and other nuclear systems.
\end{abstract}

This is an Open Access article distributed under the terms of the Creative Commons Attribution-Noncommercial License 3.0, which permits unrestricted use, distribution, and reproduction in any noncommercial medium, provided the original work is properly cited. 